providers. "Seventy-nine percent of respondents switched to a healthcare provider other than the one who initially diagnosed their herpes," said Clarke. "Sixty-four percent gave the initial provider a 'poor' rating in the area of advice on emotional issues related to herpes. This confirms what we at ASHA have heard from patients for a long time-that many physicians have great difficulty in working with patients diagnosed with herpes and other sexually transmitted diseases. Establishing personal rapport with the patient is one of the keys to successful treatment, and helping physicians become more comfortable with taking a sexual history and discussing sexuality is a goal of ASHA's planned physician education efforts."

\section{From the Centers for Disease Control}

\section{TUBERCULOSIS AMONG RESIDENTS OF SHELTERS FOR THE HOMELESS, OHIO, 1990}

During 1990, 17 cases of clinically active pulmonary tuberculosis (TB) occurred among residents of homeless shelters in three Ohio cities (Cincinnati, Columbus, and Toledo). This report summarizes the results of investigations of these cases by the Ohio Department of Health.

\section{Cincinnati}

During March 1990, health officials in Cincinnati were notified of three TB cases among residents of a 200-bed shelter for homeless adults. One of these (index case) occurred in a man with a history of alcohol abuse who died from respiratory failure and at autopsy was found to have cavitary pulmonary TB. From April through November 1990, eight additional cases of pulmonary TB were identified among residents of the shelter. Of the 11 total case-patients, seven were sputum-smear-positive, indicating potential infectiousness, and ten were culture-positive. Four case patients were known acquaintances of the index patient.

Mycobacterial isolates from the ten culturepositive patients and isolates obtained from ten persons not associated with the outbreak (controls) were sent to the Centers for Disease Control (CDC) for typing by restriction fragment-length polymorphism (RFLP). The control isolates were obtained from a convenience sample of ten persons with apparently unrelated TB cases reported during 1990 from Cincinnati and nearby counties in Ohio and Kentucky. Nine of the ten outbreak-related isolates, including the isolate from the index patient, and two control isolates had identical RFLP banding patterns. The two control isolates that shared an RFLP banding pattern with outbreak isolates were obtained from patients who, like the index patient, resided in Cincinnati and had a history of alcohol abuse.

\section{Columbus}

During March 1990, staff from a local hospital emergency room notified the public health department in Columbus of a case of sputum-smear-positive pulmonary $\mathrm{TB}$ in a resident (index patient) of a homeless shelter; TB had been diagnosed during January, but the patient had been lost to followup for two months. During those two months, he had resided in a shelter in Toledo, 135 miles north of Columbus. The public health department notified the Columbus shelter director and initiated a voluntary, city-wide TB screening and case-finding program for residents and staff of men's shelters and soup kitchens; 95\% of these facilities participated.

On average, 768 persons daily occupied the participating men's shelters and soup kitchens in Columbus. During April 24 through May 24, 1990, the city health department administered Mantoux tuberculin skin tests (five tuberculin units [TU] of purified protein derivative [PPD]) to 363 residents and 123 (69\%) of 178 staff. Of the 486 skin tests administered, 403 (83\%) were read (291 residents and 112 staff). Among 81 skin-tested residents of the shelter in which the index patient resided, $32(40 \%)$ had tuberculin skin test reactions $\geqslant 10 \mathrm{~mm}$ induration, compared with 47 (22\%) of 210 skin-tested residents of other Columbus men's shelters and soup kitchens (relative risk $=1.8$, 95\% confidence interval=1.2-2.5). Among the 27 staff members at the shelter in which the index patient resided, seven $(26 \%)$ had tuberculin skin test reactions $\geqslant 10 \mathrm{~mm}$ induration, compared with nine (11\%) of 85 staff members in other men's shelters and soup kitchens ( $\phi=.06$, Fisher's exact test, two-tailed).

Following the screening program in Columbus, vouchers for chest radiographs were issued to 95 persons with tuberculin reactions $\geqslant 10 \mathrm{~mm}$ induration (previous tuberculin status not reported) and 30 persons with previously known tuberculin reactions. Of these 125 persons, $111(89 \%)$ had radiographs and $40(32 \%)$ reported to the TB clinic for evaluation and treatment after the radiograph. Isoniazid (INH) prophylaxis was recommended for 37 of the 40 persons; $28(76 \%)$ of the 37 did not return after their initial clinic visit, eight (22\%) completed prophylaxis, and one (3\%) stopped treatment because of adverse reactions. One resident who had a tuberculin reaction $\geqslant 10 \mathrm{~mm}$ induration and two who refused a chest radiograph had culture-negative pleural TB diagnosed in June 1990. 
From May through December 1990, five additional cases of clinically active pulmonary TB were identified among residents of men's shelters and soup kitchens in Columbus. Results of investigations of these cases are pending.

\section{Toledo}

In Toledo, voluntary screening for TB was initiated at the shelter that had been visited by the index patient from Columbus and was offered to persons who resided in the shelter within ten weeks of the potential exposure. Of the 80 residents in the shelter, $20(25 \%)$ were considered to be long-term $(\geqslant 3$ months) residents; 18 of these were evaluated. Two of the 18 had histories of tuberculous infection. Mantoux tuberculin skin tests (5 TU PPD) were administered to the remaining 16 ; of the 15 skin tests that were read, four (27\%) patients had reactions $\geqslant 10 \mathrm{~mm}$ induration.

M M WR. 1991;40:869-870.

\section{FOOD AND DRUG ADMINISTRATION APPROVAL OF USE OF DIPHTHERIA AND TETANUS TOXOIDS AND ACELLULAR PERTUSSIS VACCINE}

The Immunization Practices Advisory Committee and the Committee on Infectious Disease, American Academy of Pediatrics, recommend that children routinely receive a series of five doses of vaccine against diphtheria, tetanus, and pertussis before seven years of age. ${ }^{1,2}$ The Food and Drug Administration has approved a diphtheria and tetanus toxoids and acellular pertussis vaccine (DTaP) prepared by Lederle Laboratories (Peral River, New York) and distributed as ACEL-IMUNE ${ }^{\mathrm{TM}}$ (use of trade names is for identification only and does not imply endorsement by the Public Health Service or the US Department of Health and Human Services). This vaccine is licensed only for use as the fourth and fifth doses for children who have previously been vaccinated against diphtheria, tetanus, and pertussis with three doses of whole-cell diphtheria and tetanus toxoids and pertussis vaccine (DTP) and is not licensed for the initial three-dose series in infants and children; whole-cell DTP should continue to be used for these initial doses. Whole-cell DTP continues to be an acceptable alternative for the fourth and fifth doses. DTaP is not licensed for use in children younger than 15 months of age or after the seventh birthday. The fourth dose should be given at least six months after the third dose of whole-cell DTP and is usually administered to children 15-18 months of age.1,2 A dose of DTaP may be given in the series for children ages 4-6 who have received either all four doses as whole-cell vaccine or three doses of whole-cell DTP plus one dose of DTaP; this fifth dose should be given before the child enters kindergarten or elementary school. The fifth dose in the vaccination series is not necessary if the fourth dose was given on or after the fourth birthday. 1,2

The following evidence supports the use of ACELIMUNE after the initial three-dose series of whole-cell DTP vaccine in infants:

- The immunogenicity of the antigens comprising ACEL-IMUNE when used for the fourth and fifth doses is comparable with that of the whole-cell DTP vaccine. ${ }^{3}$

Although not evaluated in a prospective study in which clinicians and investigators were blinded with respect to the vaccination status of the study subjects, the effectiveness against clinical pertussis disease of a $\mathrm{DTaP}$ vaccine manufactured and used in Japan (which contained a pertussis vaccine component identical to that in ACEL-IMUNE) has been demonstrated in children 2 years of age or older. ${ }^{4}$

- The rates of local reactions, fever, and other common systemic symptoms following receipt of ACEL-IMUNE inoculations are lower than those following whole-cell DTP vaccination. ${ }^{3}$

\section{REFERENCES}

1. Immunization Practices Advisory Committee. Diphtheria, tetanus, and pertussis: recommendations for vaccine use and other preventive measures-recommendations of the Immunization Practices Advisory Committee (ACIP). MMWR. 1991;40.

2. American Academy of Pediatrics. Report of the Committee on Infectious Diseases. Elk Grove Village, Ill: American Academy of Pediatrics; 1991

3. Blumberg DA, Mink CM, Cherry JD, et al. Comparison of an acellular pertussis-component diphtheria-tetanus-pertussis (DTP) vaccine with a whole-cell pertussis-component DTP vaccine in 17- to 24-month-old children, with measurement of 69 kilodalton outer membrane protein antibody. J Pediatr. 1990;117:46-51.

4. Mortimer EA, Kimura M, Cherry JD, et al. Protective efficacy of the Takeda acellular pertussis vaccine combined with diphtheria and tetanus toxoids following household exposure of Japanese children. Am J Dis Child. 1990;144:899-904.

M M WR. 1991;40:881-882. 